\title{
THYROID, Etc.
}

Robinson, G. Canby.-On a Cyst originating from the Ductus Thyreoglossus. "Johns Hopkins Hospital Bulletin," April, 1902.

The literature on the cysts of the anterior median cervical region is not extensive. The author gives a résumé of what little there is, and adds an interesting case from his own experience. This occurred in a coloured stevedore, aged fifty-five, who died of cerebral hæmorrhage. The tumour, of which two excellent drawings are given, proved to be a spherical unilocular cyst about $4 \frac{1}{2}$ centimetres in diameter, with a smooth, rather thin wall. It lay upon and was attached lightly to the sterno-hyoid muscles, pushing them apart about $1 \frac{1}{2}$ centimetres. Vertically it reached from the isthmus of the thyroid body to the hyoid bone, being firmly attached to the under surface of the latter. The cyst contained a glairy mucoid fluid with a mass of cellular detritus, which microscopically presented only disintegrated cells with deeply staining nuclear fragments. The walls were composed of a compact, laminated connective tissue, lined by a single layer of columnar ciliated epithelial cells. There was no thyroid tissue in any part of the tumour. Miacleod Yearsley.

\section{E A R.}

Arthur, Richard (of Sydney).-An Unusual Sequela of the Adenoid Operation. "Indian Medical Record," February 12, 1902.

In this case the operation for adenoids, which was done skilfully and with antiseptic precautions, was followed by an acute exacerbation of a chronic suppuration in the middle ear. The mastoid became involved, giving rise to thrombosis of the lateral sinus and a Bezold's abscess. Unfortunately, operation was postponed until too late, and the patient, a child of ten, succumbed.

Macleod Yearsley.

Bristow, Donald M.-Report of an Unusual Case of Deafness. "New York Medical Record," March 22, 1902.

A case which well illustrates the value of perseverance in treatment. The patient was a man aged twenty-two years. When eight years old he had an acute illness, which he was told was typhus fever; since that time he had never been able to hear with his right ear. This, although the hearing with the left was very good, inconvenienced him in the study of choral singing. He had never noticed any improvement, temporary or otherwise, in his right ear. On examination, the left ear was found to respond normally to tests and to be of normal appearance. The right membrane was retracted, the short process of the malleus being very prominent; the drum was pale, but not thickened. A 36 -inch watch could not be heard on pressure. The tuning-fork reactions were those of middle-ear deafness. The Eustachian tube was freely patent to both catheter and Politzer's douche.

Energetic inflation through the catheter gave no result. Lucae's pressure-probe was then tried without effect. The next day the patient was subjected to acoustic massage, after which the pressure-probe was again used, and finally the ear was inflated with the catheter. The immediate result was that the watch could be heard faintly on contact, and the hearing of the tuning-fork by air-conduction was improved. For three weeks the patient was treated every second day by the pressure-probe, followed by catheterization. For the first two weeks he showed slight improvement after each sitting. Then he began to 
improve more rapidly. On the eighteenth day he could hear the watch at 4 inches; on the twenty-fourth day he heard the watch at 36 inches, and, with the left ear tightly plugged, he heard every word of a conversation 20 feet away in the next room.

The author's view of the case is that the deafness was due to simple fibrous anchylosis between the ossicles, and that the bands were stretched or broken by means of the pressure-probe. Macleod Yearsley.

Burger, H.-Ear Disease and Life Insurance. "Klinische Vorträge aus dem Gebiete der Otologie und Pharyngo Rhinologie," Bd. V., Heft 4.

In this paper the relations of different ear affections to life insurance are considered. The conclusions arrived at are :

1. More attention should be paid to ear disease by insurance companies.

2. Insurance should not be effected in acute inflammations of the external and middle ear till the disease has been cured.

3. It is advisable to put off insurance in severe cases of chronic otitis externa.

4. Insurance can be effected in otitis media chronica, simplex and catarrhalis, without delay.

5. Otitis media chronica suppurativa should not be accepted if there is inflammation in the attic or mastoid, tubercle or cholesteatomata, bone disease, facial paralysis, or if there is along with the otitis vertigo, headache confined to one side, or narrowing of the meatus, which would prevent free escape of secretion.

6. Other cases of chronic suppuration do not always require to be rejected.

7. The possibility of acceptance of such cases with increased premium should be considered after examination by an otologist.

8. Permanent perforation of the tympanic membrane requires a slightly increased premium; other cases completely recovered can be accepted without.

9. Chronic cases of suppuration cured by the radical operation, in spite of great bone deficiency-viz., retro-auricular opening caused by the operation - can be accepted with increased premium.

10. Marked deafness on both sides and severe cases of vertigo should cause increased terms for acceptance.

11. It is highly desirable that the grossly neglected instruction in ear disease in the Universities should be increased and improved, to correspond with the present position of otology. Guild.

Gellé, Georges.-A Case of Mastoid Pain due to Hysteria. "Archives Internationales de Laryngologie," etc., January-February, 1902.

Dr. Gelle draws attention to the rarity of these cases (mastoid pain without auricular lesions), nine only having been recorded, as far as he can find. He describes his recent case-that of a girl, eighteen years of age, who, as a result of a severe mental shock and exposure to wet, had severe mastoid neuralgia. She was operated on, but ten days after the pain returned as bad as ever. The ear and the right side of the pharynx were hypersensitive, the patient was unable to bear the slightest touch with cotton-wool. There were none of the classic symptoms of middle-ear disease present.

Under local application of glycerine, and by suggestion the case gradually improved. Anthony McCall. 
Laurens, Georges. - Extradural Abscess, with Pachymoningitis of Middle-Ear Origin. "Annales des Maladies de l'Ureillu, du Larynx," etc., February, 1902.

The case was an old-standing one of middle-ear disease, with symptoms of antral mischief. In addition, there was stiffness of muscles of neck, vertigo, delirium, vomiting, and constipation. On opening the antrum and clearing away the granulation tissue and carious bone, the dura mater was found exposed and bathed in pus; the wound was cleansed and dressed, the symptoms gradually subsided, and the case recovered.

The dura mater was not opened, and lumbar puncture showed the cerebro-spinal fluid to be normal. Anthony McCall.

Raoult and Specker-Hamorrhage from the Ear during Typhoid Fever. " Revue Hebdom. de Laryngol.," etc., February 1, 1902.

A child, six years old, had typhoid fever. On the eleventh day of the fever it had two intestinal hæmorrhages, and on the thirteenth day an attack of epistaxis and bleeding from the right ear. The latter continued from the thirteenth to the sixteenth day, when it was replaced by suppuration. From this time the ear affection went through the course of an ordinary acute otitis media. The blood, and later the pus, escaped from the tympanic cavity through a perforation in the antero-inferior segment of the membrane.

Hæmorrhage from the ear is extremely rare in typhoid fever. Isambert in 1896 could find reports of only three cases, and Raoult and Specker have found none published since that date. Of the three cases reported, two were cases of suppuration, with more or less bloodstained pus; the third was a case of undoubted hæmorrhage, but the blood came not from the tympanum, but from a bulla on the external surface of the membrana tympani, in this respect resembling many cases of hæmorrhage reported in influenza. Arthur J. Hutchison.

Reik, H. 0.-Catarrhal Otitis Media (Non-suppurative) as a Factor in the Etiology of Facial Paralysis. "Johns Hopkins Hospital Bulletin," April, 1902.

The author believes that non-suppurative otitis media is a neglected factor in facial paralysis. The points he particularly lays stress upon are as follows :

1. If exposure to cold in one way or another is the cause of most cases of facial paralysis, it probably acts most commonly through the production of an otitis media, whereby the nerve becomes involved either in a direct extension of the inflammatory process to its exposed sheath, or through pressure upon it of an exudate.

2. This being admitted, the form of treatment which offers the most rapid and satisfactory result is a paracentesis of the tympanic membrane, to free the cavity of its abnormal contents.

3 . We should never in any case of facial paralysis neglect to examine the ear. Macleod Yearsley.

Thomas.-A Case of Otitic Pyamia without Phlebitis; Suppurative Choroiditis of the Opposite Eye. "Revue Hebdom. de Laryngol., etc., January 11, 1902.

The patient was a man, aged forty-nine, who after a chill began to suffer from severe earache. On examination (July 11), there was found 
obstruction of the Eustachian tube, acute non-suppurative otitis media, acute mastoiditis, with bulging of the posterior wall of the external auditory meatus. The mastoid operation was at once proposed, but refused; therefore a paracentesis was done. No pus came away. The treatment then advised consisted in ice to the mastoid and instillations of carbolized glycerine. The patient was not seen for a month. When he returned there was a purulent discharge from the ear, the posterior wall of the meatus was swollen and very tender, and signs of mastoiditis persisted. August 17: Mastoid antrum was opened. Bone dense, eburnated. Antrum full of granulation tissue; little or no pus. Progress satisfactory till August 31, when pain commenced in the wound, and great tenderness was found at the inner end of the external meatus. Osteitis or osteo-phlebitis of the inner end of the meatus was diagnosed, and the author was about to recommend a second interference-viz., a complete Stacke operation, when on September 7 conjunctivitis appeared in the left eye.

The left eye became painful and prominent. At first retrobulbar abscess was suspected, but later the condition was found to be one of suppurating choroiditis.

Signs of meningitis soon appeared, and patient died on September 28. No post-mortem examination was made. The author considers that the course of events in this case was mastoid abscess, giving rise to osteitis, thence infection of the eye by the blood-stream, then infection of the meninges from the eye.

Arthur J. Hutchison.

\section{REVIEWS.}

The Accessory Sinuses of the Nose, their Surgical Anatomy, and the Diagnosis and Treatment of their Inflammatory Affections. By A. Logan Turner, M.D., F.R.C.S. Eảin., Surgeon for Diseases of Ear and Throat, Deaconess Hospital, Edinburgh. Forty plates, 81 figs., pp. 211. William Green and Sons, Edinburgh, 1901.

This volume, the result of some years of careful work on the part of Dr. Turner, has for its fundamental basis the lectures delivered by him at the Royal College of Surgeons, Edinburgh, in 1898, on "The Illumination and Surgical Anatomy of the Skull."

The maxillary sinus and outer nasal wall form the subject of the first chapter, and we find a lucid description of the nasal fossæ included, together with a short account of the development of the maxillary aircell. According to the investigations of the author, the three molar teeth are those which are most constantly directly under this cavity, and consequently should be selected as the sites for perforating through the alveolus to enter the antrum. When entering the antrum through its nasal wall we are told that that part of the bone lying immediately below the attachment of the inferior turbinate is not only preferable from the fact that the bone is here the thinnest, but also as by choosing this spot we are less liable to miss a very small antrum. The usual dimensions are given, and also all variations at present known. The frontal sinus is next considered, and at considerable length, commencing with a short sketch of its development. The direction, course, size, and variations of the ostium frontale are entered into most minutely, 